08

\title{
Динамика теплового излучения, сопровождающего конденсацию паров вольфрама в газообразном и сверхтекучем гелии
}

\author{
(C) А.В. Карабулин, ${ }^{1,2}$ М.И. Кулиш, ${ }^{1}$ В.И. Матюшенко, ${ }^{3}$ М.Е. Степанов ${ }^{1}$ \\ ${ }^{1}$ Институт проблем химической фризики РАН, \\ 142432 Черноголовка, Россия \\ ${ }^{2}$ Объединенный институт высоких температур РАН, \\ 125412 Москва, Россия \\ ${ }^{3}$ Филиал Федерального исследовательского центра химической физики им. Н.Н. Семенова РАН, \\ 142432 Черноголовка, Россия \\ e-mail: avkarabulin@gmail.com
}

Поступило в Редакцию 3 сентября 2020 г.

В окончательной редакции 13 ноября 2020 г.

Принято к публикации 14 ноября 2020 г.

Методом пирометрических измерений исследованы процессы остывания продуктов импульсной лазерной абляции вольфрамовой мишени в вакууме, гелиевом газе и в жидком сверхтекучем гелии. Показано, что количественные измерения характеристик тепловой эмиссии позволяют выяснить характерные особенности механизмов процессов конденсации наноструктур в данных средах. На основании полученных данных рассчитаны сечения излучающих частиц. Показано, что в вакууме излучают преимущественно субмикронные частицы, в то время как в сверхтекучем гелии - наночастицы. Полученные результаты подтверждаются данными электронной микроскопии.

Ключевые слова: импульсная лазерная абляция, вакуум, конденсация, жидкий сверхтекучий гелий.

DOI: 10.21883/JTF.2021.04.50629.258-20

\section{Введение}

Образование субмикронных и наночастиц в результате импульсной лазерной абляции (ИЛА) представляет собой сложный процесс, интересный для исследования, как с прикладной, так и с фундаментальной точек зрения. ИЛА является одним из наиболее простых и универсальных методов, дающим возможность получать наночастицы практически из любых материалов, при этом, в отличии от разнообразных химических методов, с чистой поверхностью, не загрязненной какими-либо реагентами. На форму, размеры, химический состав и прочие параметры получаемых в результате абляции продуктов оказывают влияние различные факторы: длина волны лазера, его мощность и длительность импульса, состояние поверхности мишени, а также характеристики среды, в которой проводится абляция [1-3]. Как правило, продуктами абляции являются сферические кластеры, чье распределение по размерам обычно описывается логнормальной функцией, а размеры варьируются от единиц до сотен нанометров [1]. В отдельных случаях путем применения дополнительных факторов (например, в сильных магнитных полях) возможно получение продолговатых наночастиц [4]. Но существует среда, абляция металлов в которой неизменно приводит к образованию тонких (диаметром 2-10 nm, в зависимости от металла), длинных (аспектное отношение доходит до 1000) нанопроволок [5]. Этой средой является квантовая жидкость сверхтекучий гелий (Не II). В работе [5] был предложен механизм образования нанопроволок, основанный на ряде свойств Не II, в частности, на наличии в нем квантованных вихрей, способных захватывать любые примеси. Этот механизм включает в себя концентрирование наночастиц металла, являющихся первичным продуктом абляции, в квантованных вихрях и их плавление при взаимном слиянии внутри вихря за счет тепла, выделяющегося при уменьшении поверхностной энергии.

Одним из способов исследования процессов образования нанопроволок является изучение динамики теплового излучения продуктов абляции, сопровождающего процесс синтеза наноструктур в Не II, и его сравнение с динамикой теплового излучения кластеров в вакууме, где механизм образования наночастиц гораздо проще и значительно лучше изучен.

Исследованию теплового излучения наночастиц посвящен целый ряд экспериментальных работ [6-18]. Исследовались как одномерные наноструктуры (углеродные нанотрубки и нанопроволоки), так и наночастицы и кластеры сферической формы. В этих работах исследовались такие особенности теплового излучения наноматериалов как излучательная способность наночастиц, эффективность потерь энергии на тепловое излучение, диапазон применимости закона Стефана-Больцмана с уменьшением размера частиц. Из приведенных работ следует, что тепловое излучение наночастиц описывается формулой Планка с излучательной способностью, зависящей от длины волны: $\varepsilon \sim \frac{1}{\lambda}$. При этом считается, что частицы находятся в состоянии оптически тонкого ансамбля в отличие от случая крупных частиц, когда 
излучение происходит из расположенного вблизи поверхности частиц скин-слоя.

Задачей настоящей работы являлось изучение влияния сверхтекучести гелия ${ }^{4} \mathrm{He}$ на процессы конденсации продуктов импульсной лазерной абляции вольфрамовой мишени путем регистрации теплового излучения, сопровождающего этот процесс, и сравнения полученных данных с излучением нанокластеров в вакууме и газе. Понимание механизма роста нанопроволок в сверхтекучем гелии может помочь в создании методики получения квазиодномерных объектов с заданными свойствами.

\section{1. Постановка эксперимента}

Схема экспериментальной установки показана на рис. 1. Заливной гелиевый криостат OptCryo оборудован сапфировыми окнами, через которые проводилась абляция расположенной внутри криостата мишени, и регистрировалось тепловое излучение, сопровождающее коагуляцию металла. При работе в сверхтекучем гелии температура поддерживалась постоянной путем откачки паров гелия и составляла $T \approx 1.2 \mathrm{~K}$ (что соответствует давлению паров менее 1 Torr). Эксперименты в вакууме осуществлялись при комнатной температуре и давлении $10^{-2}$ Torr, в газообразном гелии - при комнатной температуре и атмосферном давлении.

Абляция осуществлялась с помощью сфокусированного излучения импульсно-периодического ИК лазеpa (Nd:LSB-лазер с диодной накачкой (STA-01-05W, Standa)) с энергией импульса $0.1 \mathrm{~mJ}$ и полушириной импульса $0.4 \mathrm{~ns}$. Рабочая длина волны лазера $1064 \mathrm{~nm}$, частота повторения импульсов $50 \mathrm{~Hz}$. Лазерный пучок

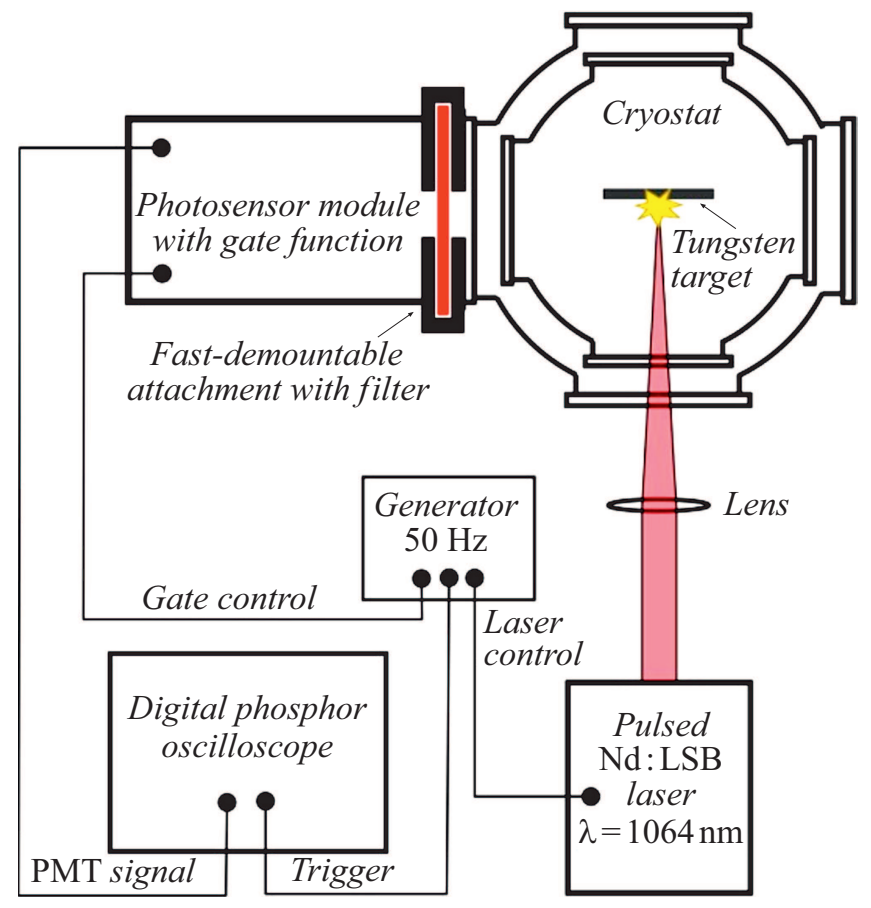

Рис. 1. Схема экспериментальной установки. фокусировался длиннофокусной $(F=280 \mathrm{~mm})$ линзой на плоскую поверхность мишени в пятно диаметром около $100 \mu \mathrm{m}$. Таким образом, плотность мощности на поверхности мишени составляла $W \sim 3 \cdot 10^{9} \mathrm{~W} / \mathrm{cm}^{2}$.

Оптическая регистрация проводилась фоточувствительным модулем H11526-01-NN (Hamamatsu), расположенным на расстоянии $100 \mathrm{~mm}$ от места абляции. Модуль был выполнен на основе фотоумножителя (РМТ) с эффективным диаметром $8 \mathrm{~mm}$, оснащенного внутренним затвором. Управление включением/выключением затвора модуля выполняется управляющими сигналами c TTL (transistor-transistor logic) уровнями со временем переключения порядка $100 \mathrm{~ns}$. Сигнальный разъем фоточувствительного модуля подключался к согласованному входному сопротивлению канала осциллографа TDS 7054 (Tektronix). Полоса пропускания осциллографа составляла $500 \mathrm{MHz}$, время нарастания сигнала фотоумножителя 0.57 ns. Осциллограф, лазер и фоточувствительный модуль управлялись генератором с выходными сигналами TTL-уровней. Генератор запускал схему регистрации с частотой $50 \mathrm{~Hz}$.

В начале цикла работы установки закрывался затвор фотоумножителя и подавался сигнал на запуск лазера. В случае попадания на фотокатод ФЭУ мощного импульса лазерного излучения система регистрации на длительное время выходит из области линейного преобразования светового потока в напряжение и происходит искажение сигнала даже после окончания лазерного импульса. С целью предотвращения этого фотоумножитель включался через $280 \mathrm{~ns}$ после воздействия лазерного импульса на мишень. Величина времени задержки была подобрана экспериментально. Задержка в $280 \mathrm{~ns}$ оказалась достаточной для исключения влияния излучения лазера. Лазерный импульс совмещен с нулем времени на регистрируемых осциллограммах. Величина импульсного выходного тока фотоумножителя не превышала $30 \mathrm{~mA}$, что соответствовало линейной области работы РМТ по току в импульсном режиме. Осциллограммы записывались в режиме 128-кратного усреднения сигнала. При обработке сигналов учитывался уровень постоянной засветки окружающим светом. Для этого при механически перекрытом луче лазера записывался нулевой сигнал, который затем вычитался из полезного сигнала. Коэффициент усиления фотоумножителя для спектральных измерений устанавливался равным $\approx 5 \cdot 10^{6}$.

Перед фотокатодом располагались сменные интерференционные фильтры. Для достоверного описания реального спектра излучения использовались семь фильтров от 400 до $700 \mathrm{~nm}$ с шагом $50 \mathrm{~nm}$ (продукция фирмы Thorlabs). Полная ширина полосы пропускания фильтров на полувысоте (FWHM) составляла $10 \mathrm{~nm}$. В спектральных расчетах везде принималось, что полоса пропускания фильтров бесконечно узкая. Кроме того, при описании излучения АЧТ учитывался тот факт, что размер излучающих частиц в нашем случае существенно меньше длин волн наблюдаемого света. 
При абляции в сверхтекучем гелии весь процесс конденсации происходит на расстоянии 1-2 mm от поверхности мишени [19]. В вакууме скорость разлета медленной компоненты при абляции лазером, сходным с используемым в настоящей работе, составляет порядка $100 \mathrm{~m} / \mathrm{s}$ [20], поэтому за время $200 \mu \mathrm{s}$ наночастицы перемещаются на расстояние $L=20 \mathrm{~mm}$ и не покидают поле зрения ФЭУ. Таким образом, в обоих случаях излучение собирается со всего объема, в котором происходит конденсация.

Следовательно, можно считать, что полученные зависимости отражают кинетику полной высвечиваемой при конденсации энергии.

Продукты конденсации оседали на дно экспериментальной ячейки, куда помещались обычно используемые в просвечивающей электронной микроскопии (ПЭМ) сеточки диаметром $3 \mathrm{~mm}$, покрытые перфорированной углеродной пленкой. После разогрева криостата до комнатной температуры образцы исследовались в электронном микроскопе JEOL JEM-2100.

Геометрия эксперимента (взаимное расположение мишени, регистрирующего оборудования, лазерного пучка) оставалась неизменной при проведении абляции во всех средах.

Объектом исследования был выбран вольфрам, у которого из-за высокой температуры плавления интенсивность свечения, сопровождающая синтез, была максимальной и достаточной для ее уверенной регистрации в интервале времени до $200 \mu \mathrm{s}$. Для металлов с меньшими температурами плавления интенсивность сигнала заметно меньше, так как с уменьшением температуры в рассматриваемой области длин волн сигнал экспоненциально уменьшается. Это приводит к более короткому времени регистрации сигнала. Так, например, для легкоплавкого индия регистрация излучения была возможна только в течение $10 \mu \mathrm{s}$.

\section{2. Определение цветовой температуры излучения}

В настоящей работе регистрировались импульсные сигналы, меняющиеся в большом динамическом диапазоне. Для настоящей работы была разработана методика калибровки, позволяющая совместить измерение импульсных сигналов с калибровкой по источнику постоянного излучения.

Калибровка спектральной чувствительности фотоумножителя производилась с помощью стабилизированной лампы накаливания SLS201L (Thorlabs) и дополнительно проверялась по сигналу от эталонной ленточной вольфрамовой лампы накаливания СИРШ-8.5-200-1. Для лампы SLS201L распределение излучения по спектру считалось соответствующим формуле Планка с температурой 2796 К, у лампы СИРШ-8.5-200-1 ток питания выбирался так, чтобы температура накаливаемой ленты составляла $2700 \mathrm{~K}$. Методика калибровки по ленточной лампе и зависимость степени черноты вольфрама от длины волны бралась из [21]. Калибровочные лампы являются непрерывными источниками света, а для постоянного тока режим линейной области работы фотоумножителя ограничен величиной $100 \mu \mathrm{A}$. При калибровке излучение ламп ослаблялось в $\sim 10^{5}$ раз нейтральными фильтрами ND30A и ND20A (Thorlabs) и после прохождения через сменные интерференционные фильтры регистрировалось на увеличенном до $R=10 \mathrm{k} \Omega$ нагрузочном сопротивлении фотоумножителя. При этом фиксировалась величина усредненного постоянного выходного сигнала, которая для всех спектральных интервалов не превышала по току $100 \mu \mathrm{A}$. При калибровке делались поправки на слабые спектральные зависимости пропускания нейтральных фильтров ND30A и ND20A, приведенные в паспортных данных фильтров. Величины сигналов калибровки по спектральным каналам использовались для определения спектральной чувствительности системы регистрации, необходимой при расчетах цветовой температуры в процессе абляции и коагуляции металлов.

Сигнал калибровки $S_{\text {calib }}$ связан с температурой $T$ лампы в каждом спектральном канале следующим выражением:

$S_{\text {calib }}(\lambda)=B(\lambda) \operatorname{Tr}_{N D 20 A}(\lambda) \operatorname{Tr}_{N D 30 A}(\lambda) \varepsilon(\lambda, T) \frac{1}{\lambda^{5}} \frac{1}{e^{\frac{h c}{\lambda k T}}-1}$,

где $\lambda$ - центральная длина волны спектрального канала, $B(\lambda)$ - коэффициент пропорциональности, $\operatorname{Tr}_{N D 20 A}(\lambda)$ и $\operatorname{Tr}_{N D 30 A}(\lambda)$ - коэффициенты пропускания нейтральных фильтров, $\varepsilon(\lambda, T)$ - степень черноты вольфрама. Лампа SLS201L откалибрована так, что $T=2796 \mathrm{~K}$ и степень черноты можно считать постоянной, например $\varepsilon(\lambda, T)=1$. Для лампы СИРШ-8.5-200-1 $T=2700 \mathrm{~K}$ и $\varepsilon(\lambda, 2700 \mathrm{~K})<1$ определялась по данным [21]. Величина $B(\lambda)$ зависит от многих параметров: спектральной ширины канала, геометрии сбора света от источника, спектральной чувствительности ФЭУ, величины нагрузочного сопротивления ФЭУ.

Из приведенного выражения для сигнала калибровки определялись величины $B(\lambda)$ для каждого канала, так как все остальные входящие величины известны. Для определения цветовой температуры излучения наночастиц экспериментальные сигналы для каждого из семи каналов первоначально нормировались на определенную по калибровке величину $B(\lambda)$ для соответствующего канала. Далее проводилось усреднение сигналов по 100 точкам для снижения уровня шума. Полоса регистрируемых частот исследуемого сигнала снижалась при усреднении до $F_{\max }=1.07 \mathrm{MHz}$. В каждом сигнале устранялся сдвиг нуля, возникающий при осциллографической записи. Подготовленные таким образом данные в каждой временной точке фитировались методом наименьших квадратов распределением излучения черного тела по формуле Планка с подбором двух параметров - температуры и масштабного коэффициента. По приведенному алгоритму определялась временная зави- 
симость температуры и зависимость сечения излучения частиц от времени.

\section{3. Результаты и обсуждение}

Характерные для каждой среды особенности излучения кластеров проявляются во всех рассмотренных аспектах свечения: в динамике интенсивностей свечения, в динамике цветовых температур, в поведении сечений излучения. Эти особенности наглядно прослеживаются в зависимостях интенсивности излучения от времени, представленных на рис. 2 для длины волны $700 \mathrm{~nm}$ (длина волны $\lambda=700 \mathrm{~nm}$ близка к максимуму спектральной интенсивности для АЧТ при наблюдаемых температурах; ход кривых для других регистрируемых длин волн практически одинаков).

Кривая 1 , соответствующая излучению в вакууме, в начальной части кривой расположена ниже кривой 2 (излучение в Не II), хотя, как видно из рис. 3, случай вакуума соответствует более высоким цветовым температурам. С другой стороны, в начальные моменты интенсивность излучения в вакууме 1 и в газе 3 совпадает. Из этих данных можно сделать предположение: первичные продукты абляции в вакууме и газе схожи между собой и отличаются от таковых в Не II. Кроме того, следует отметить темп снижения интенсивности излучения: в атмосфере газа интенсивность излучения быстро спадает, почти столь же быстро она спадает в Не II, и относительно медленный спад регистрируется для кластеров в вакууме.

Представляется разумным предположение, что в вакууме и газе источниками излучения служат крупные частицы (далее микрочастицы), тогда как в Не II источниками излучения являются малые частицы (далее наночастицы). Исходя из этого, в настоящей работе для обработки экспериментальных данных была применена

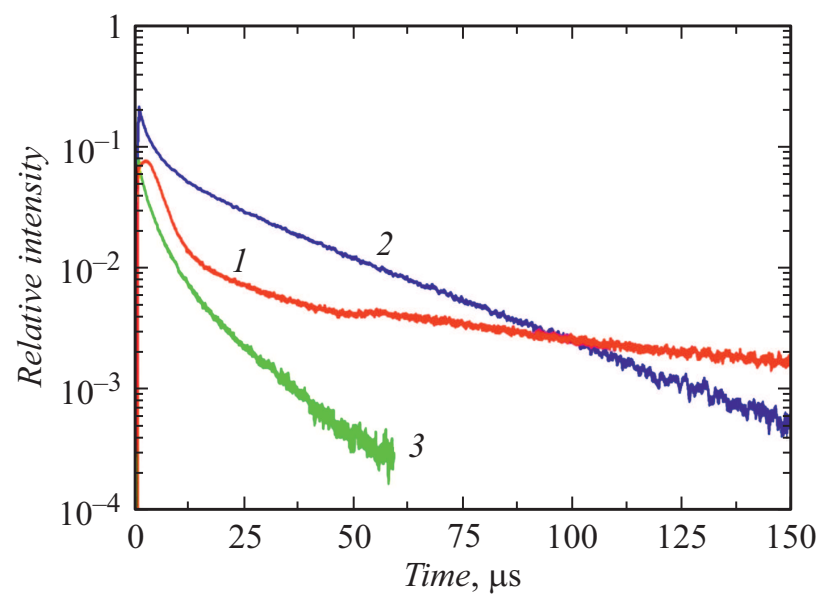

Pис. 2. Зависимость интенсивности излучения от времени для длины волны $700 \mathrm{~nm}$ в вакууме (1), в сверхтекучем гелии (2) и в газообразном гелии при нормальных условиях (3).

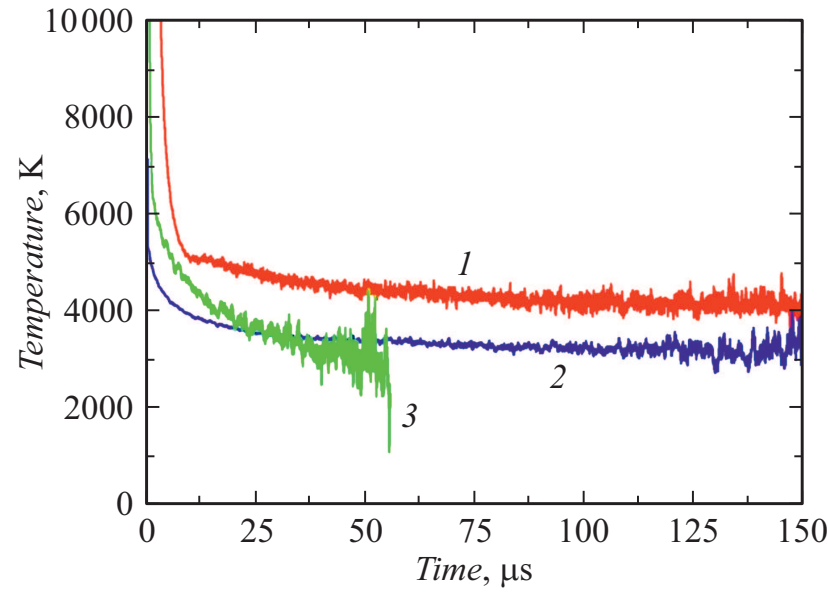

Рис. 3. Температура излучения кластеров вольфрама в процессе его конденсации в вакууме $(1)$, в сверхтекучем гелии (2) и в газообразном гелии при нормальных условиях (3).

подгоночная зависимость:

$$
I(\lambda, T)=S \frac{1}{\lambda^{6}} \frac{1}{e^{\frac{h c}{\lambda k T}}-1}
$$

в случае наночастиц и

$$
I(\lambda, T)=S \frac{1}{\lambda^{5}} \frac{1}{e^{\frac{h c}{\lambda k T}}-1}
$$

для микрочастиц, где дополнительный множитель $1 / \lambda$ возникает из-за учета излучательной способности наночастицы [22], а $S$ пропорционально суммарному сечению излучения ансамбля частиц. На рис. 3 приведена зависимость температур излучения кластеров вольфрама при абляции мишени в вакууме 1, в сверхтекучем гелии 2 и гелиевом газе при атмосферном давлении 3. При абляции в вакууме начальный участок до $10 \mu \mathrm{s}$ характеризуется высокой температурой с максимумами интенсивности на длинах волн менее $400 \mathrm{~nm}$. В этих условиях метод цветового определения температуры имеет низкую точность. Достоверное определение температур начинается после $10 \mu \mathrm{s}$. При разлете испаренного вещества в вакуум расширяющийся поток состоит из двух фракций - газообразного вещества и частиц [20]. До $t \sim 10 \mu \mathrm{s}$ газообразная составляющая, расширяясь, быстро охлаждается и далее наблюдается излучение частиц вольфрама.

Ход наблюдаемой температуры при разлете в сверхтекучий гелий 2 начинается сразу с $T \sim 5000 \mathrm{~K}$. Это вызвано интенсивным взаимодействием испаренного вещества с гелием. Интересно рассмотреть то, как температурные зависимости процессов в вакууме и в сверхтекучем гелии соотносятся с температурой плавления объемного вольфрама $T_{m}$. Как видно из рис. 3 , в случае вакуума температура на всем протяжении превышает $T_{m}$, тогда как в Не II, наоборот, стабилизируется ниже этого уровня. Объяснение такого различия можно найти, если учесть особенности плавления наночастиц. Согласно [23], для 

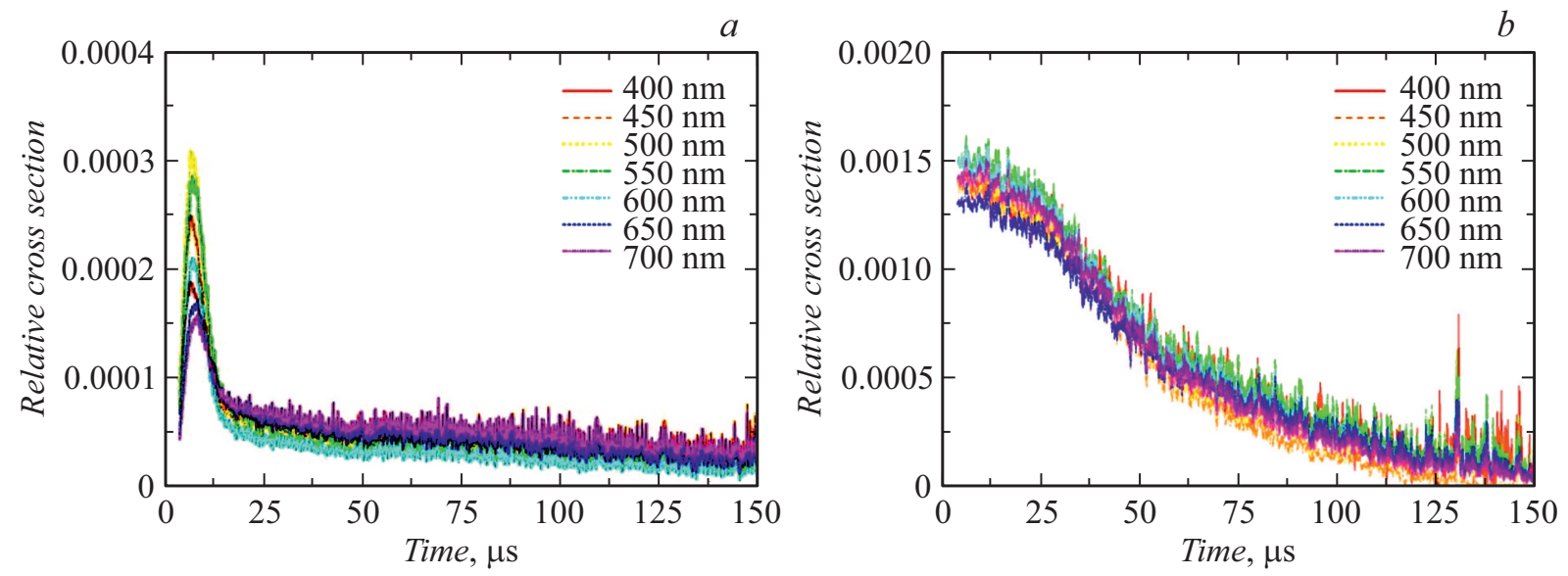

Рис. 4. Зависимость сечения излучения частиц от времени для различных длин волн в вакууме $(a)$ и в сверхтекучем гелии $(b)$.

достаточно больших частиц $(R>100 \mathrm{~nm})$, когда доля поверхностных атомов становится малой, теплофизические характеристики таких частиц приближаются к объемному случаю. Для остывания столь больших частиц, соответствующих в нашем случае абляции в вакууме, трудно ожидать, что частицы успеют термализоваться до температур плавления за времена наблюдения, поскольку их крупный размер препятствует быстрому остыванию. В сверхтекучем же гелии, напротив, мы имеем дело с большим количеством малых нанопроволок или их фрагментов, которые обеспечивают быстрое остывание ввиду повышенной способности к радиационному отводу тепла, при этом оставаясь в зоне видимости ФЭУ. Согласно [23], для вольфрамовых нанопроволок наших размеров (длина фрагментов $10 \mathrm{~nm}$ при радиусе $1 \mathrm{~nm}$ ) ввиду большой доли поверхностных атомов температура плавления должна снижаться по сравнению с объемным случаем, и прямая оценка предсказывает снижение на $15 \%$ до температуры $3200 \mathrm{~K}$, что и наблюдается в эксперименте (рис. 3). Таким образом, предположение об излучающих микронных частицах в вакууме (газе) и излучающих наночастицах в Не II находит дополнительное подтверждение.

Зависимость от времени при разлете в атмосферу гелия 3 характеризуется заметно более быстрым спадом температуры, вызванным теплообменом с гелием при давлении много большем, чем в случае кривой 2 .

Зависимость сечения излучения частиц $S$ от времени для различных длин волн показана на рис. 4 для разлета в вакуум и в сверхтекучий гелий. Кривые приведены в относительных единицах, но в одинаковом масштабе. Сечение излучения частиц практически одинаково для всех длин волн, что подтверждает принятую модель излучения черного тела. Для разлета в вакуум (рис. $4, a$ ) после $10 \mu$ s величина сечения на порядок меньше случая конденсации в сверхтекучем гелии и слабо меняется от времени. Если принять, что масса полученных единичным лазерным импульсом частиц не зависит в первом приближении от среды, что подтверждается микроскопией мишеней после абляции, то различия в эффективных сечениях можно объяснить различием в распределении массы вещества по ансамблю наночастиц. В вакууме доля массы в излучающих частицах меньше, чем в случае (рис. $4, b$ ), и излучение происходит с поверхности относительно крупных частиц. В Не II масса распределена между большим количеством частиц малого размера, эффективно излучающих со всего объема ввиду оптической прозрачности ансамбля для видимого диапазона, что обеспечивает большую величину эффективного сечения в начале кривой. На временах $\sim 25 \mu \mathrm{s}$ происходит смена механизма коагуляции частиц. Согласно механизму роста нанопроволок, предложенному в работе [5], частицы сплавляются в квантованных вихрях, образуя нанопроволоки, и разогретые области занимают только часть нанопроволок, что выражается в уменьшении сечения на кривой (рис. $4, b$ ).

Если учесть результаты электронной микроскопии образцов (рис. 5), а именно то, что в вакууме образуются сферические кластеры диаметром до $500 \mathrm{~nm}$, а в Не II металлические сетчатые структуры, в которых индивидуальные проволоки имеют диаметр примерно $2 \mathrm{~nm}$, то излучающие свет малые частицы в случае Не II можно отождествить с нанопроволоками, а большие частицы в вакууме - с шарами большого диаметра.

Аналитический вид зависимости температуры частиц в вакууме от времени (1 на рис. 3) может быть получен из расчета охлаждения частиц за счет теплового излучения:

$$
C_{v} \frac{d T}{d t}=-\varepsilon \sigma S T^{4}
$$

где $\sigma$ - постоянная Стефана-Больцмана, $\varepsilon-$ степень черноты вольфрама, $C_{v}=4.5 \cdot 10^{6} \mathrm{~J} /\left(\mathrm{m}^{3} \cdot \mathrm{K}\right)$ - теплоемкость единицы объема вольфрама.

Решение этого уравнения приводит к выражению для скорости охлаждения микрочастицы:

$$
\frac{1}{T^{3}}=\frac{9 \varepsilon \sigma}{r C_{v}} t=K t
$$

где $r$ - характерный радиус кластера. 
$a$

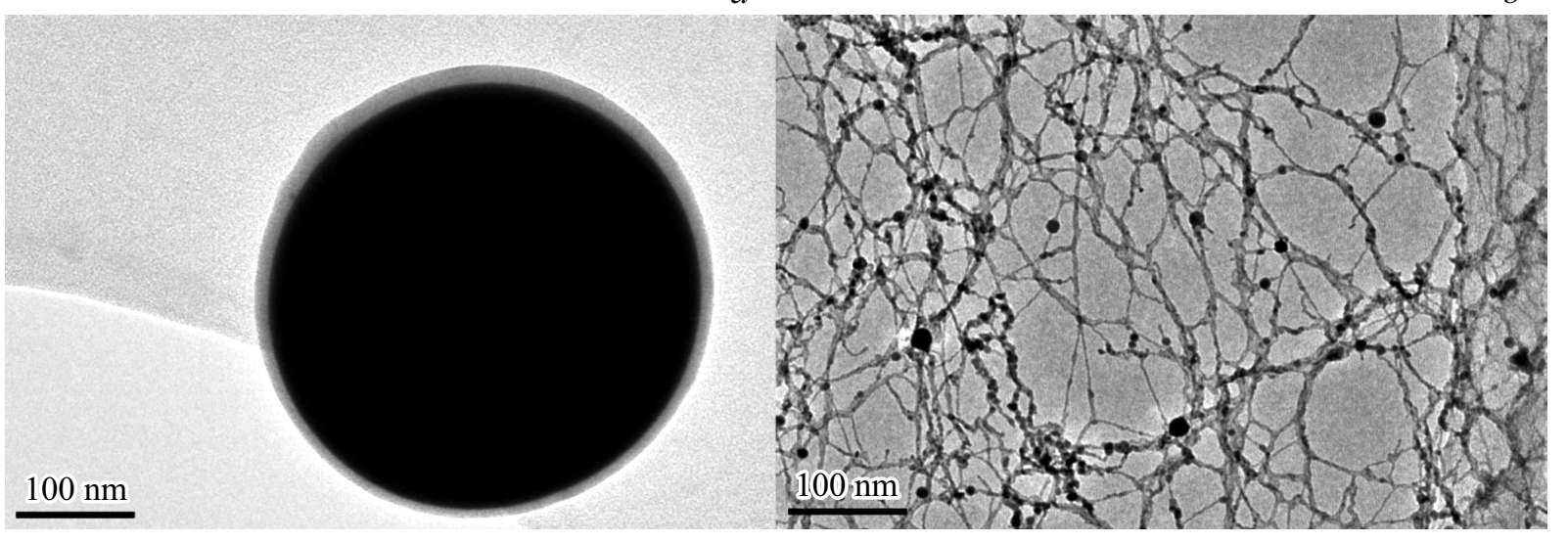

$b$

Рис. 5. Электронная микроскопия образцов, полученных абляцией вольфрамовой мишени в вакууме $(a)$ и в сверхтекучем гелии $(b)$.

Из экспериментальных данных определяем величину $K=6 \cdot 10^{-8} 1 /\left(\mathrm{K}^{3} \cdot \mathrm{s}\right)$. Отсюда определяется характерный радиус частиц: $r=0.6 \mu \mathrm{m}$.

Экспериментальная кривая зависимости температуры излучения от времени при абляции в вакуум и расчетная зависимость охлаждения частиц за счет теплового излучения представлены на рис. 6 . Видно, что температура излучения при $t>10 \mu$ s практически определяеся радиационным охлаждением.

Зависимость температуры излучения от времени при абляции в сверхтекучий гелий (кривая 2, рис. 3) может быть описана с учетом особенностей излучения наночастиц и влиянием коагуляции на ход зависимости температуры. Согласно [24], запишем выражение для спектральной мощности излучения частицы:

$$
P_{\omega}=c \sigma_{a b s}(\omega) U(\omega) / 4
$$

где $U(\omega)$ - спектральная плотность излучения черного тела [25]:

$$
U(\omega)=\frac{\hbar \omega^{3}}{\pi^{2} c^{3}\left(e^{\frac{\hbar \omega}{k T}}-1\right)} .
$$

Сечение поглощения электромагнитных волн $\sigma_{a b s}[26]$ для сферического кластера, размеры которого малы по сравнению с длиной волны излучения $\lambda \sim \frac{c}{\omega}$ :

$$
\sigma_{a b s}=\pi r^{2} \frac{r}{\lambda} f(\omega)
$$

Функция $f(\omega)$ зависит от действительной и мнимой частей диэлектрической проницаемости вещества $\varepsilon^{\prime}, \varepsilon^{\prime \prime}$ как [26]:

$$
f(\omega)=\frac{12 \varepsilon^{\prime \prime}}{\left(\varepsilon^{\prime}+2\right)^{2}+\varepsilon^{\prime \prime 2}} .
$$

Данные для диэлектрической проницаемости вольфрама приведены в работе [27] и для вольфрама в нормальных условиях рассчитанная величина $f(\omega)$ меняется от 0.03 до 0.3 в оптическом диапазоне частот.

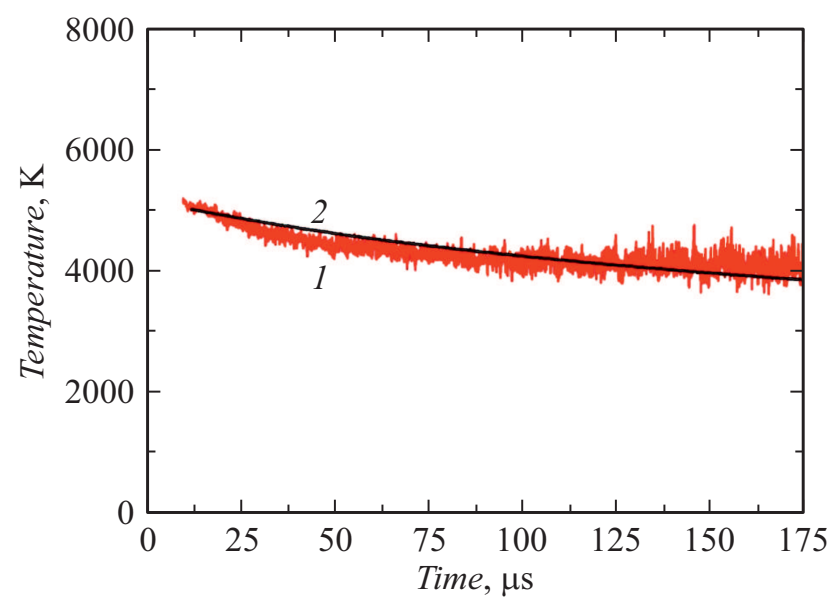

Рис. 6. Экспериментальная кривая (1) зависимости температуры излучения частиц от времени при абляции $W$-мишени в вакууме и расчетная зависимость (2) охлаждения частиц за счет теплового излучения.

При переходе к длинам волн получим

$$
P_{\lambda}=\sigma_{a b s}(\lambda) \frac{2 \pi h c^{2}}{\lambda^{5}} \frac{1}{e^{\frac{h c}{k c T}}-1} .
$$

Для ансамбля частиц сумма сечений:

$$
\Sigma \sigma_{a b s}(\lambda)=\frac{3}{4} V \frac{f(\lambda)}{\lambda}
$$

где $V$ - объем всех кластеров.

Мощность, излучаемая ансамблем частиц во всем спектральном диапазоне, равна

$$
I=\int_{0}^{\infty} P(\lambda) d \lambda=\frac{3}{4} V f\left(\lambda_{m}\right) \frac{2 \pi(k T)^{5}}{h^{4} c^{3}} 24 \xi(5),
$$

где $\xi(5)$ - дзета функция Римана, $f\left(\lambda_{m}\right)$ - некоторое среднее значение функции $f(\lambda)$. Решаем уравнение ра- 


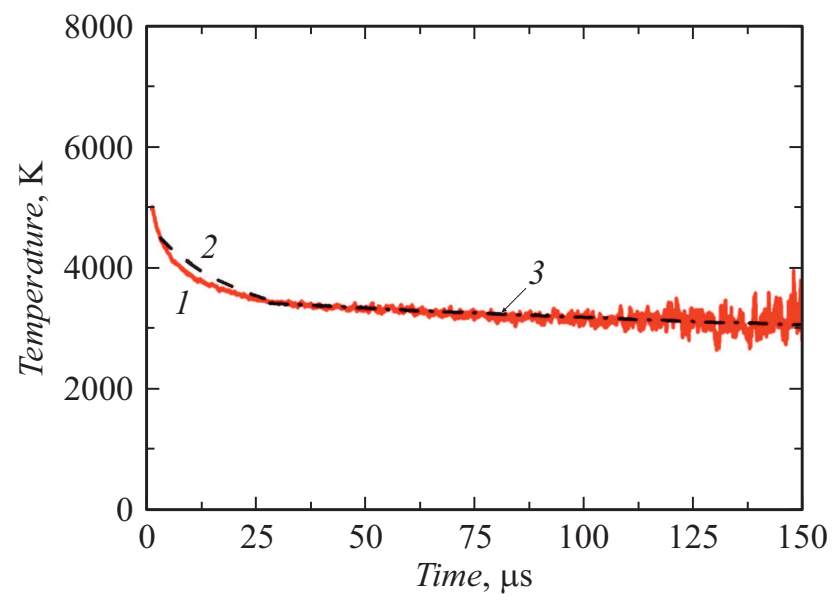

Рис. 7. Экспериментальная кривая (1) зависимости температуры излучения частиц от времени при абляции $W$-мишени в сверхтекучем гелии и расчетные зависимости $(2,3)$ охлаждения частиц.

диационного остывания

$$
C \frac{d T}{d t}=-I,
$$

где $C$ - теплоемкость объема $V$. Получаем

$$
\frac{1}{T^{4}}-\frac{1}{T_{0}^{4}}=A\left(t-t_{0}\right)
$$

где

$$
A=3 f\left(\lambda_{m}\right) \frac{2 \pi k^{5}}{h^{4} c^{3}} 24 \zeta(5) \frac{1}{C_{v}} .
$$

Здесь $C_{v}$ - теплоемкость единицы объема вещества. Величина $f(\lambda)$ в видимой области меняется от 0.03 до 0.3 для холодного вещества. Для вещества при температуре $T \sim 4000 \mathrm{~K}$ величина $f(\lambda)$ должна быть больше, так как удельное сопротивление вольфрама растет с температурой.

Для оценки сверху примем $f\left(\lambda_{m}\right)=1$. Тогда

$$
A=3 f\left(\lambda_{m}\right) \frac{2 \pi k^{5}}{h^{4} c^{3}} 24 \xi(5) \frac{1}{C_{v}}=1.0 \cdot 10^{-11} \mathrm{~s}^{-1} \cdot \mathrm{K}^{-4},
$$

где теплоемкость $C_{v}=4.5 \cdot 10^{6} \mathrm{~J} \cdot \mathrm{m}^{-3} \cdot K^{-1}$ рассчитана для вольфрама при температуре $T \sim 3700 \mathrm{~K}$.

Экспериментально величина $A$ для излучения в Не II была определена для двух характерных временных интервалов 0-30 и 30-150 $\mu \mathrm{s}$, в которых механизм остывания частиц различен. В первом случае она составляет $A_{1}=1.75 \cdot 10^{-10} \mathrm{~s}^{-1} \cdot \mathrm{K}^{-4}$, что близко к экспериментальным данным [22], по которым $A=2.15 \cdot 10^{-10} \mathrm{~s}^{-1} \cdot \mathrm{K}^{-4}$. Во втором интервале темп остывания заметно меньше (с константой $A_{2}=0.326 \cdot 10^{-10} \mathrm{~s}^{-1} \cdot \mathrm{K}^{-4}$ ). В этом случае уменьшение темпа остывания связано с разогревом участков нанопроволок при сплавлении их в квантованных вихрях Не II.

Экспериментальная кривая 1 зависимости температуры излучения частиц от времени при абляции $W$-мишени в сверхтекучем гелии с расчетными зависимостями 2 и 3 охлаждения частиц представлены на рис. 7.

\section{Заключение}

Показано, что измерение интенсивности излучения ансамбля микро- и наночастиц вольфрама в процессе абляции металлической мишени позволяет определить поведение температуры частиц в различных условиях эксперимента и выяснить характерные особенности механизмов процессов конденсации наноструктур в данных средах.

В случае вакуума и газа зависимость температуры частиц от времени при абляции $W$-мишени при $t>10 \mu \mathrm{s}$ полностью определяется радиационным охлаждением. При этом из характера излучения следует, что излучение происходит с поверхности относительно крупных (субмикронных и микронных) частиц. В случае Не II, несмотря на низкую температуру среды (менее 2K) и уникально высокую теплопроводность сверхтекучей жидкости, наблюдается уменьшение скорости остывания по сравнению с абляцией в вакууме, а из анализа сечений излучающих частиц можно сделать вывод, что источником излучения является большое количество частиц малого размера, эффективно излучающих со всего объема ввиду оптической прозрачности ансамбля для видимого диапазона. Это находится в согласии с данными электронной микроскопии продуктов абляции, которые позволяют предполагать, что малыми излучающими центрами в Не II выступают нанопроволоки (или их разогретые фрагменты), являющиеся специфическим продуктом, характерным только для абляции в Не II, что связано со свойствами сверхтекучего гелия - его рекордной теплопроводностью и наличием квантованных вихрей. А большие излучающие центры в вакууме и газообразном гелии - это в основном шарообразные кластеры, в целом характерные для абляции в этих средах.

\section{Финансирование работы}

Работа выполнена при финансовой поддержке гранта РНФ № 18-19-00620.

\section{Конфоликт интересов}

Авторы заявляют, что у них нет конфликта интересов.

\section{Список литературы}

[1] N.G. Semaltianos. Crit. Rev. Solid State Mater. Sci., 35 (2), 105 (2010). DOI: 10.1080/10408431003788233

[2] H. Zeng, X.-W. Du, S.C. Subhash, S.A. Kulinich, S. Yang, J. He, W. Cai. Adv. Funct. Mater., 22 (7), 1333 (2012). DOI: $10.1002 / \mathrm{adfm} .201102295$ 
[3] A. Kanitz, M.-R. Kalus, E.L. Gurevich, A. Ostendorf, S. Barcikowski, D. Amans. Plasma Sources Sci. Technol., 28 (10), 103001 (2019). DOI: 10.1088/1361-6595/ab3dbe

[4] G.A. Shafeev, I.I. Rakov, K.O. Ayyyzhy, G.N. Mikhailova, A.V. Troitskii, O.V. Uvarov. Appl. Surf. Sci., 466, 477 (2019). DOI: 10.1016/j.apsusc.2018.10.062

[5] Е.Б. Гордон, А.В. Карабулин, В.И. Матюшенко, В.Д. Сизов, И.И. Ходос. ЖЭТФ, 139 (139), 1209 (2011). [Е.В. Gordon, A.V. Karabulin, V.I. Matyushenko, V.D. Sizov, I.I. Khodos. J. Exp. Theor. Phys., 112 (6), 1061 (2011).]

[6] Y. Fan, S.B. Singer, R. Bergstrom, B.C. Regan. Phys. Rev. Lett., 102 (18), (2009) DOI: 10.1103/PhysRevLett.102.187402

[7] H. Zeng, C. Yang, J. Dai, X. Cui. J. Phys. Chem. C, 112 (11), 4172 (2008). DOI: 10.1021/jp711607n

[8] M. Sveningsson, M. Jönsson, O.A. Nerushev, F. Rohmund, E.E.B. Campbell. Appl. Phys. Lett., 81 (6), 1095 (2002). DOI: $10.1063 / 1.1498493$

[9] S. Osswald, K. Behler, Y. Gogotsi. J. Appl. Phys., 104(7), 074308 (2008). DOI: 10.1063/1.2980321

[10] D.R. Ward, N.J. Halas, D. Natelson. Appl. Phys. Lett., 93 (21), 213108 (2008). DOI: 10.1063/1.3039060

[11] S. Amoruso, R. Bruzzese, N. Spinelli, R. Velotta, M. Vitiello, X. Wang. Europhys. Lett. EPL, 67 (3), 404 (2004). DOI: $10.1209 / \mathrm{epl} / \mathrm{i} 2004-10080-2$

[12] J. Costa, P. Roura, J.R. Morante, E. Bertran, J. Appl. Phys., 83 (12), 7879 (1998). DOI: 10.1063/1.367965

[13] T. Donnelly, J.G. Lunney, S. Amoruso, R. Bruzzese, X. Wang, X. Ni. J. Appl. Phys., 108 (4), 043309 (2010).

DOI: $10.1063 / 1.3475149$

[14] Ю.В. Мартыненко, Л.И. Огнев. ЖТФ, 75 (11), 130 (2005). [Yu.V. Martynenko, L.I. Ognev. Tech. Phys., 50 (11), 1522 (2005).] DOI: $10.1134 / 1.2131968$

[15] M. Rosenberg, R.D. Smirnov, A.Y. Pigarov. J. Phys. Appl. Phys., 41 (1), 015202 (2008). DOI: $10.1088 / 0022-3727 / 41 / 1 / 015202$

[16] А.Н. Магунов. Спектральная пирометрия (Физматлит, M., 2012).

[17] S.S. Harilal, N. Farid, A. Hassanein, V.M. Kozhevin. J. Appl. Phys., 114 (20), 203302 2013). DOI: 10.1063/1.4833564

[18] Е.В. Гуренцов, А.В. Еремин, С.А. Мусихин. ЖТФ, 89 (8), 1200 (2019). DOI: 10.21883/JTF.2019.08.47891.2335

[19] E.B. Gordon, M.I. Kulish, A.V. Karabulin, V.I. Matyushenko. Low Temp. Phys., 43 (9), 1086 (2017).

[20] S. Amoruso, R. Bruzzese, X. Wang, N.N. Nedialkov, P.A. Atanasov. J. Phys. Appl. Phys., 40 (2), 331 (2007). DOI: $10.1088 / 0022-3727 / 40 / 2 / 008$

[21] В.И. Малышев. Введение в экспериментальную спектроскопию (Наука, М., 1979).

[22] U. Frenzel, U. Hammer, H. Westje, D. Kreisle. Z. Phys. D, 40, 108 (1997).

[23] W.H. Qi, M.P. Wang. Mater. Chem. Phys., 88(2-3), 280 (2004). DOI: 10.1016/j.matchemphys.2004.04.026

[24] B.M. Smirnov. Phys. Uspekhi, 36 (7), 592 (1993).

[25] M. Planck. Ann. Phys., 4 (553), 1 (1901).

[26] L.D. Landau, E.M. Lifshitz. Electrodynamics of Continuous Media (Pergamon Press, Oxford, 1960)

[27] W.S.M. Werner, K. Glantschnig, C. Ambrosch-Draxl. J. Phys. Chem. Ref. Data, 38 (4), 1013 (2009).

DOI: $10.1063 / 1.3243762$ 